\title{
Hallazgos numismáticos en la villa romana de «La Pila», Altea (Alicante)
}

\author{
Manuel Abad VAREla*
}

Presentamos en este pequeño estudio un conjunto de monedas recogidas sobre el terreno, en la villa romana de Altea (Alicante). Tres de éstas, además del material arqueológico, nos sirvieron para adelantar en su día una cronología de la misma ${ }^{1}$. Ahora damos a conocer, junto con esas tres otros cuatro hallazgos más.

Se encuentra el yacimiento en la Partida de La Pila, en Altea, próximo al margen izquierdo del río Algar (fig. 1.)

\section{ANTECEDENTES}

Se contaba con algunos antecedentes arqueológicos sobre la zona, aunque no todos muy claros, que podrian estar, por su proximidad, en relación con la villa, como vamos a ver. Pero estos son los primeros hallazgos numismáticos de los que se tiene noticia en Altea.

Con el surgir de los ilustrados, aquellos humanistas del siglo XVIII, se busca con ansia por todo el país, incluida esta zona alicantina, todo tipo de inscripciones en excavaciones o paredes de edificios antiguos. Cualquier

* Departamento de Prehistoria e Historia-Antigua, UNED.

- Para más información sobre esta villa se puede consultar: "Una villa romana en La Pila, Altea (Alicante)", Altea (Boletin de Información Municipal), año IX, n. ${ }^{\circ} 99$, agosto, 1987 , págs. 15-17; O la comunicación, con el mismo título, al $X I X$ Congreso Nacional de Arqueología, Castellón de la Plana (diciembre de 1987), (en prensa). 


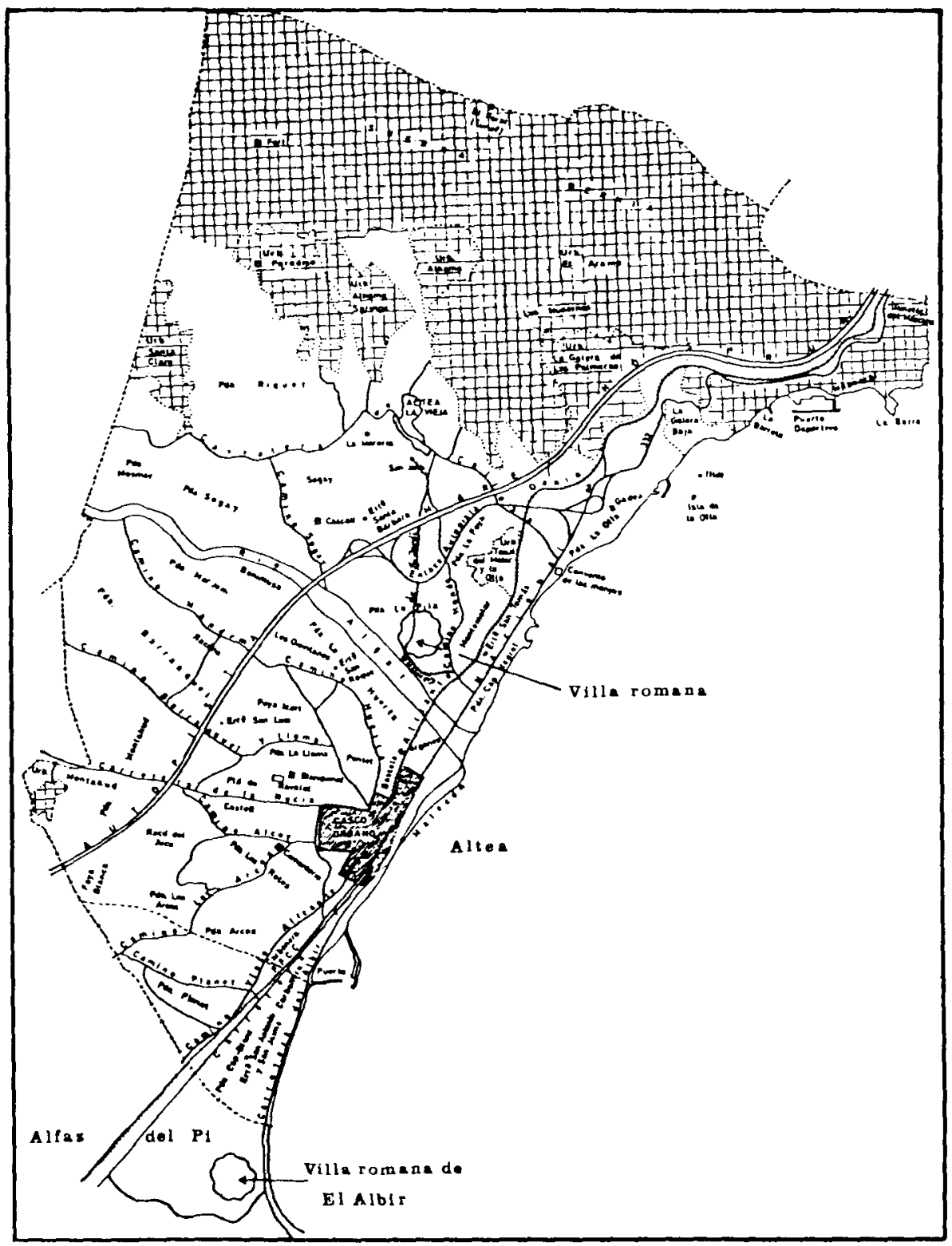

Fig. 1. Plano de Altea con sus partidas y caminos. 
hallazgo era celebrado, incluso los numismáticos por los que sentían un gran interés ${ }^{2}$. Un ejemplo lo tenemos en Antonio Valcarcel Pío de Saboya, quien en 1773 habia escrito ya su pequeña obra: Medallas de la Colonias, Municipios, y Pueblos Antiguos de España hasta hoy no publicadas ${ }^{3}$ Y no nos cabe ninguna duda de su interés por estos temas, pues en una carta dirigida a Fernando José de Velasco en 1774 confesaba: "Mi pasión en este estudio (numismático) es tanta que no tengo embarazo en viajar 20 leguas para una medalla" ${ }^{4}$. Con este entusiasmo por conseguir antigüedades pasaría por Altea algo después, en torno al 1778. Pero sólo nos hablará, entonces, de Cap Negret que se encuentra muy cerca de la misma y próxima a La Pila. Allí nos dice se encontraban trozos de inscripciones, barros saguntinos, "monedas" y fragmentos de urnas y de ánforas" ${ }^{5}$. No hay otra información más explícita sobre hallazgos de monedas en sus proximidades. Tampoco en su obra, indica el origen o circunstancias del hallazgo de las piezas que describe ${ }^{6}$, únicamente señala la pertenencia. El $P$. Ribelles también nos dejó en un manuscrito en 1808 la noticia de la aparición en el término de La Pila de un fragmento de lápida romana que se encontraba entonces en el archivo de la iglesia parroquial ${ }^{7}$. Noticia que recogerá también en su obra José Sanchís y Sivera ${ }^{8}$.

\footnotetext{
2 A. Mestre Sanchis: Humanismo y critica histórica en los ilustrados alicantinos. Alicante, 1980.

${ }_{3}^{3}$ Publicada en Valencia, y avalada en su inicio con una carta de D. Gregorio Mayáns y Siscár. En realidad con este trabajo de 21 págs. y una lámina, únicamente trataba de completar algo más la obra del P. Enrique Florez: Medallas de las colonias, municipios y pueblos antiguos de España, Madrid, $1 .^{\circ}$ vol. en 1757 y $2 .^{\circ}$ vol. en 1758 . El $3 .^{\circ}$ lo publicaria en 1773 con el fin de incluir todas las correcciones y novedades que le fueron aportando. Antonio Valcarcel dice que "La mayor parte de las noticias que incluye este escrito, estaban destinadas para que el autor citado ( $P$. Florez) las colocara en su obra; pero habiendo llegado tarde, y recogido yo posteriormente otras, no he querido privar al público de este veneficio".

${ }_{4}^{4}$ F. J. Velasco y Zevallos: (Noticias y correspondencia numismática, Vol III:) Carta $1{ }^{a}$ de Antonio Valcárcel Pio de Saboya y Espinola a don Fernando de Velasco desde Orihuela, 10 de abril de 1774 pág. $1{ }^{a}$, reverso. Ms. B. N., N. ${ }^{\circ} 2538$.

${ }^{5}$ A. VAlCARCEL PIO DE SABOya: Inscripciones y antigüedades del Reino de Valencia. tomo VIII en Memorias de la R.A. de la $H^{a}$, con anotaciones e ilustraciones de Antonio Delgado, Madrid, 1852, pág. 20.

${ }^{6}$ A. ValCarCel: Medallas de las colonias, municipios, $y$...

7 RIBELLES: "Colección de todas las lápidas romanas del Reino de Valencia publicadas e inéditas, distribuidas por orden alfabético de los pueblos en donde se han descubierto o existe", según nos dió a conocer J. SANCHís y Sivera: La diócesis Valentina. Estudios históricos, Valencia, 1920 , pág. 33 , donde dice que en esta fecha estaba inédito, y sivió de fuente fuente para otros muchos trabajos manuscritos. Desconozco si se llegó a publicar o si existe todavía dicho manuscrito. Lo que está claro es que Sanchis lo utilizó, como se ve en la nota 6.

8 SANChIS y Sivera, J.: La diócesis... pág. 41. Donde afirma: "Lo vió Ribelles en 1808 , y nos es imposible descifrarlo". La presenta con el $n^{\circ} 17$ y describe sus letras: D /HII ARVS.
} 
Pero todos estos autores no nos describen ningún hallazgo numismático en La Pila o proximidades. Aunque sí está claro que se conocian en la zona antecedentes romanos, el mismo P. Ribelles daba, en otro escrito suyo, gran importancia al "distrito de La Pila». Al debatir el tema, hoy suficientemente aclarado, de la Altheia, capital de los olcades, dice "estuvo en donde existen todavía sus ruinas, más abajo de Altea la Vieja en el distrito fronterizo al cabo Negret, conocido por el nombre de Pila" ${ }^{9}$.

A partir de aquí, no tenemos ninguna otra noticia sobre esta partida por parte de Marcelino Gutiérrez del Caño, a pesar de su afición por los temas numismáticos, ya que conocemos escribió a principios de siglo una obra titulada: Elementos de numismática española ${ }^{10}$; ni que sepamos, de Francisco Martínez Martínez ${ }^{11}$. Sólo en 1981, José G. Morote Barberá vuelve a referirse a esta partida, aunque no añade nada nuevo salvo que "en La Pila existen vestigios romanos y visigodos" ${ }^{12}$. Y finalmente, en 1983, Ramón Lloréns Barber, siguiendo al Sr. Morote, nos dice en su Diccionario de Altea y sus cosas, al hablar de La Pila, que en el siglo XVIII se encontraron restos de poblados visigodos y romanos ${ }^{13}$.

Por todo lo expuesto, parece que en la partida de La Pila se habia recogido un fragmento de lápida, por lo menos, y se tenía conocimiento de la existencia de restos arqueológicos, que al P. Bartolomé Ribelles le hicieron sospechar fuesen los cimientos de una antigua ciudad. Pero todo ello sin determinar claramente su localización, lo cual nos hace suponer que no se hace mención a este entorno donde se encuentra asentada la villa, sino a otras zonas donde hay indicios arqueológicos y quizás sean los mismos a los que se refiere el Sr. Morote.

De esta forma, vemos que la villa romana existente en el término o partida de La Pila, en Altea, no se conocía, ni se había dado a conocer

9 “Reseña de diferentes antigüedades y memorias valencianas anteriores a la dominación cartaginesa". Tomado de M. GUTIÉRREZ DEL CAÑo: Monografía histórica de la Villa de Altea, Valencia, 1920. He utilizado la edición facsímil, Altea-Benisa, 1980, pág. 14. Lo cita: Ms. del año 1808 publicado en 1911, pág. 120.

${ }^{10}$ Sabemos por la obra de J. Somoza: De la numismática en Asturias, Gijón, 1984, pág. 54, que el ilustre Don Marcelino Gutiérrez del Caño, perteneciente al cuerpo de Archiveros Bibliotecarios y Arqueólogos, tenía a principios de siglo su obra manuscrita de cuatrocientas páginas terminada y lista para imprimir. Desconocemos si se llegó a publicar.

"F. Martinez Martinez: "Antigüedades de Altea Capnegret", Saitabi IV, 1943, págs. 22-26. Aunque habla de las proximidades de La Pila, no señala ningún hallazgo en ella.

12 J. G. MORETE BARBERA: "Recordando una inscripción encontrada en el siglo XVIII", Altea, n. ${ }^{\circ} 24,1981$.

${ }_{13}$ R. LloRens Barber: Diccionario de Altea y sus cosas, Altea, 1983, pág. 251. Agradezco a mi buen amigo Ramón Lloréns, tan apasionado por las cosas de Altea, todas sus atenciones. 
ninguna moneda procedente de Altea. Por esta razón, los hallazgos monetarios que damos a conocer ahora nos pueden aportar algún dato más para el conocimiento de la villa y de la zona.

\section{ESTUDIO}

Ya conocemos todos la ayuda que en estos casos representa el hallazgo de monedas para la datación de un yacimiento. En éste nos ha permitido confirmar la supervivencia del mismo, estando de acuerdo con los restantes objetos recogidos.

Conviene hacer notar las dificultades que a menudo encierra la localización de yacimientos arqueológicos en la zona de Levante, pues el cultivo en terrazas ha hecho que muchos de éstos desaparezcan, queden ocultos o desperdigados sus restos. Esta villa presenta algunas de estas dificultades de destrozo, ocultamiento y dispersión, no obstante, el paso del arado ha facilitado la recogida de los materiales en superficie.

Las monedas, encontradas hasta el momento, son las siguientes:

\section{DESCRIPCIÓN}

N..$^{\circ}$

Antonino Pío (152-153)

Av. ANTONINVS AVGVG. PIVS P. P. TR. P. X: VI.

Busto laureado a derecha.

Rv. INDulGENtia avg cos IIII

Indulgencia sentada a dcha.

Ex. SC

Ceca: Roma

AE Sextercio

P. $24,50 \mathrm{~g}$.

M. $34 \mathrm{~mm}$.

Gr. $4 \mathrm{~mm}$.

Ej. 6

Conserv. M (foto 1) 

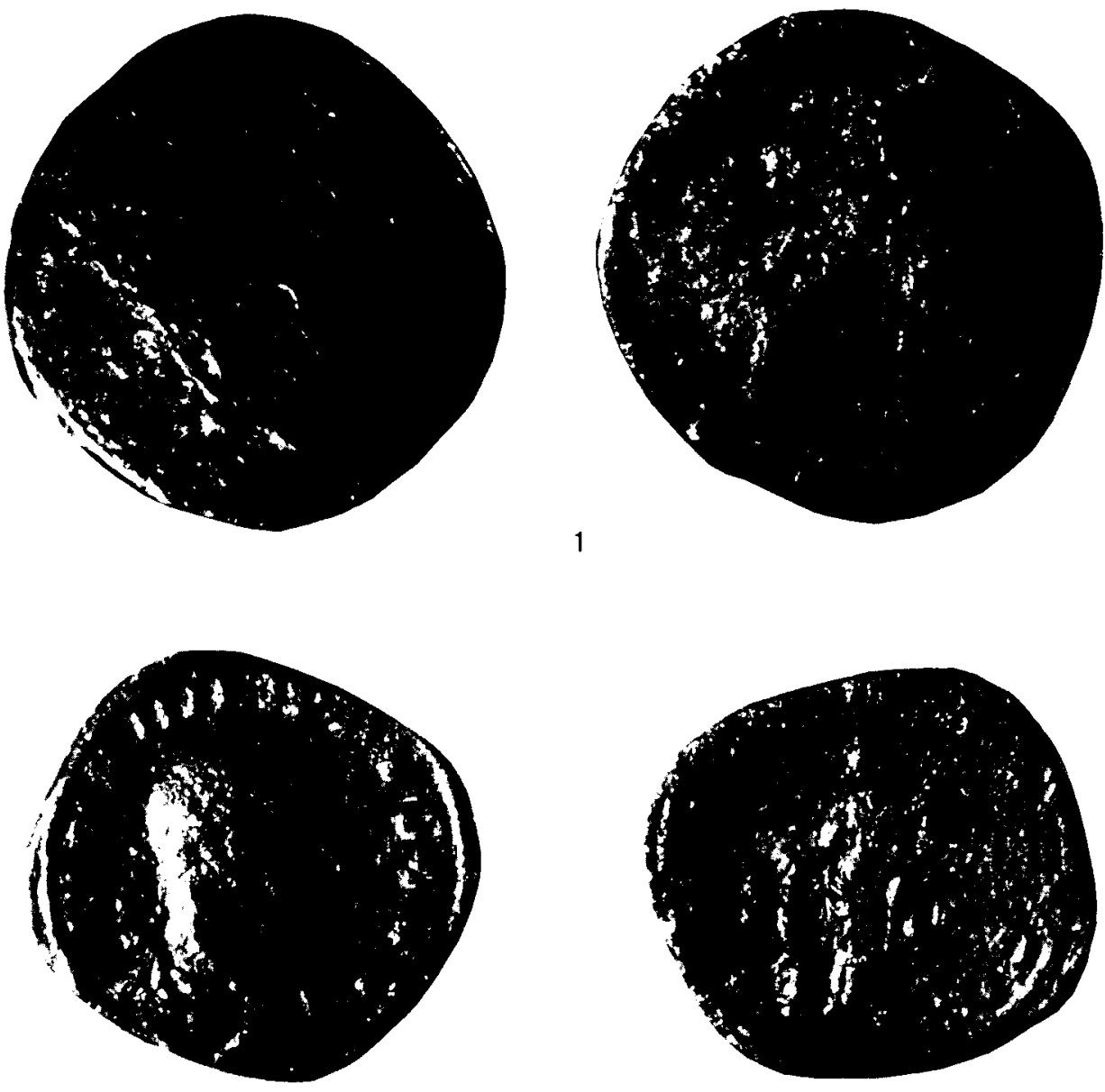

Fig. 2. 
Bib. de referencia: MATtingly, $H_{\text {.: }}$ Coins of the roman empire in the British Museum. Vol. IV n. 1.923 y foto 18 de lámina 46. Con la variante AVGVG.

N. ${ }^{\circ} 2$

Filipo I (224-249)

Av. IMP PHILIPPVS AVG

Busto laur. a dcha.

Rv. ANNONA AVGG en el campo S C

Annona de pie a iz.

Ceca: Roma

AE Sextercio

P. 21, 65 grs.

M. $28 \mathrm{~mm}$.

Gr. $4 \mathrm{~mm}$.

Ej. 12

Conserv. R. (foto 2)

Bib. de referencia: RIC n. ${ }^{\circ} 168$ a; Cohen V, n. 29.

N. ${ }^{\circ} 3$

Gallieno (261)

Av. galliENVS AVG

Cabeza radiada a dcha.

Rv. FORTVna reDVX

Fioruna de pie, sostiene timón en su dcha. y cornucopia en izq.

En campo: - S.I

Ceca: Roma $4 .^{\text {a }}$ emisión. $5 .^{a}$ oficina.

AE Antoniniano

P. $2,67 \mathrm{~g}$.

M. $19 \mathrm{~mm}$

Gr. 1,5

Ej. 5

Conserv. R (foto 3 )

Bib. de referencia: RIC n. ${ }^{\circ}$ 193; ROBERTSON n. ${ }^{\circ}$ 58, lám. 12.

N. ${ }^{\circ} 4$

Claudio II divinizado (270)

Av. DIVO CLAVDIO

Cabeza radiada a dcha.

Rv. CONSECRATIO

Altar

Ceca: Roma

AE Antoniniano

P. $1,80 \mathrm{~g}$. 
M. $15 \mathrm{~mm}$.

Gr. $1,8 \mathrm{~mm}$.

Ej. 11

Conserv. R (foto 4)

Bib. de referencia: RIC n. ${ }^{\circ}$ 261; Robertson n. ${ }^{\circ}$ 6, lám. 21.

N. 5

Constantino I (322-323)

Av. CONSTAN TINVS AVG

Busto con casco de penacho a lo largo y coraza.

Rv. BEATA TRAN QVILLITAS

Altar, con la inscripción VO/TIS/XX, sobre él globo, y encima tres estrellas.

Ex. *PTR*

Ceca: Tréveris $1 .^{a}$ oficina

$\mathrm{AE}$ nummus

P. $3,210 \mathrm{~g}$.

M. $19 \mathrm{~mm}$.

Gr. $2 \mathrm{~mm}$.

Ej. 6

Conserv. MB (foto 5)

Bib. de referencia: RIC n. ${ }^{\circ} 368$

N. ${ }^{\circ} 6$

Constancio II (351-354)

Av. D. N. CONSTAN TIVS P. F. AVG

Cabeza con diadema simple y roseta a derecha y "paludamentum». Rv. FEL TEMP REPARATIO

Infante alanceando a un jinete cayendo en tierra.

Ex. SMHA

Ceca: Heraclea $1 .^{\text {a }}$ oficina.

$\mathrm{AE} 3$

P. 2,62 grs.

M. $17 \mathrm{~mm}$.

Gr. $1,5 \mathrm{~mm}$.

Ej. 12

Conserv. R. (foto 6)

Bib. de referencia: LBRC n. ${ }^{\circ} 1900$; RIC n. 90.

N. ${ }^{\circ} 7$

Constancio II (355-361)

Av. ¿d $n$ constan tivs $p f$ avg?

Busto diademado a dcha.

Rv. FEL TEMP reparatio 
Infante alanceando a un jinete cayendo en tierra.

Ex. SMNA?

Ceca: Nicomedia $1 .^{\text {a }}$ oficina

AE 4

P. $2 \mathrm{~g}$.

M. $13 \mathrm{~mm}$.

Gr. $2 \mathrm{~mm}$.

Ej. 12

Conserv. $M$ (foto 7)

Bib de referencia: LRBC $n .{ }^{\circ} 2.311 ; R I C n .{ }^{\circ} 96$

Observ. Corresponde su clasificación a $A E 3$, sin embargo es un $A E$ 4.

Al ser un conjunto tan pequeño pocas son las conclusiones a las que podemos llegar. No obstante, observamos que cubren el espacio cronológico que va desde el año 152 al 361 d.C., estando más representados los siglos III y IV. Esto es lógico, teniendo en cuenta que la superficie actual está más próxima a los estratos de este período, aunque también, el terreno, está revuelto a causa de la construcción de terrazas $\mathrm{y}$ las plantaciones de almendros.

Si comparamos estos numismas con el resto de los hallazgos sueltos de la zona levantina, observamos que, utilizando la obra de Pere Pau Ripollés como punto de referencia ${ }^{14}$, son bastante frecuentes los hallazgos numismáticos de estos emperadores. Siguiendo su periodización, en el grupo.VIII (69-192 d.C.), las monedas de Antonio Pio se encuentran, en relación con las restantes de este periodo, en una proporción del 8,08 por 100 , estando en el $5 .^{\circ}$ puesto sobre $15^{15}$. Las de Filipo I y Claudio II son dos de las que aparecen más frecuentemente, sobre todo la de Claudio II divinizado. En el período IX (193-305) se clasifican con una asiduidad del 4,79 por 100 y 19,18 ocupando respectivamente los puestos $4 .^{\circ}$ y $2 .^{\circ}$ entre un número de 43 representaciones ${ }^{16}$. Respecto al grupo del siglo Iv, las de Constantino I son aquellas que aparecen mayor número de veces, un 21,92 por 100 y las de Constancio II un 11,53 por 100 , ocupando ambas, dentro de este período, los puestos respectivos $1 .^{\circ}$ y $3 .^{\circ}$, entre $24^{17}$.

${ }^{14}$ P. P. Ripollés: La circulación monetaria en las tierras valencianas durante la antigüedad. Barcelona 1980, pág. 158.

15 ldem., ídem pág. 158.

${ }^{16}$ Idem., ídem págs. 161-2.

17 Idem., ídem págs. 167-9. 

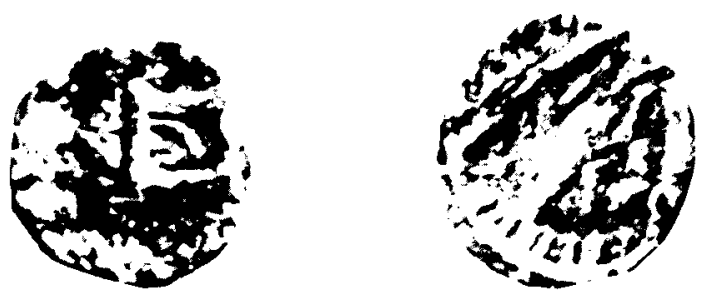

$\forall$
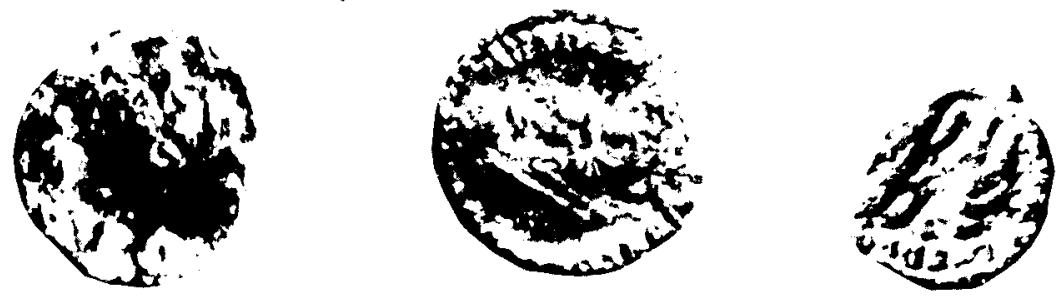

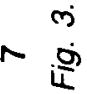
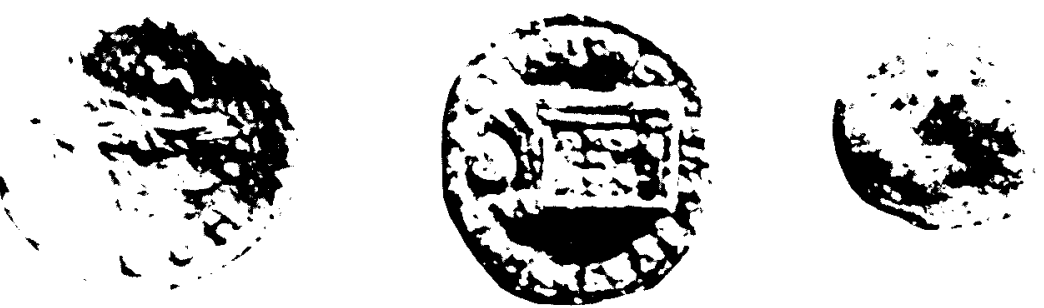

$m$
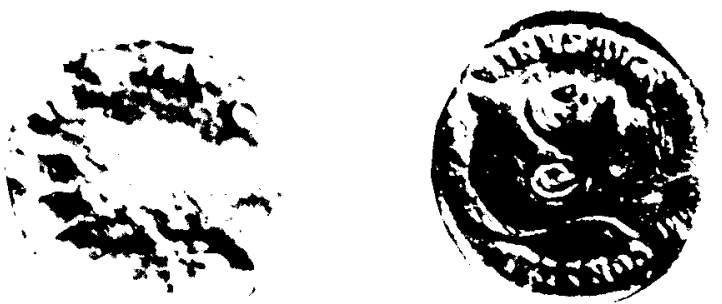
Como podemos comprobar, estos hallazgos en la zona levantina entran dentro de lo general.

En cuanto al estudio de las cecas sólo podemos fijarnos en las del siglo IV, que son las únicas que nos pueden aportar alguna información. Relacionándolas con el resto de la circulación monetaria de la zona, vemos que son relativamente frecuentes. Predominan las cecas orientales sobre las occidentales (dos de una), pero es una tendencia ya anunciada por Pere Pau Ripollés ${ }^{18}$. También es verdad que con tres ejemplares poco se puede deducir, no obstante, la tendencia se manifestaba a partir de 52,38 por 100 para las occidentales y 47,61 por 100 para las orientales. La representación de la occidental corresponde a la ceca de Tréveris que es la más frecuente, puesto que en la zona ocupa un 33,33 por 100 entre las cecas occidentales. De las dos orientales la de Nicomedia es la que resulta más extraña en la zona, por el momento, mientras que la de Heraclea su presencia es habitual. No establecemos más relaciones con el resto de la Península por ser una muestra poco relevante.

\section{CONCLUSIONES}

Por todo lo que hemos expuesto, la representación numismática, extraída hasta el momento de la villa romana, corresponde a la tónica general de los hallazgos aislados que se han venido produciendo en la zona de Levante.

Si utilizamos su valor cronológico para señalar el período de vigencia de la villa, vemos que nos marca una existencia ininterrumpida desde mediados del siglo II hasta finales del IV, como nos demuestra el desgaste de la monedas de Constancio II, últimas del período.

Ateniéndonos a los materiales arqueológicos recogidos en superficie, podemos afirmar que la misma tuvo vida, por lo menos, desde el siglo I hasta época musulmana. Ello lo atestiguan la cerámica campaniense B, la sigillata aretina lisa, la sudgálica, la hispánica y la sigillata clara, ade-

${ }^{18}$ Idem., ídem pág. 168. 
más los trozos de lucernas y cerámica vidriada, así como otros fragmentos de vidrio, mármol, etc.

Confrontadas las dos fuentes, numismática y arqueológica, vemos que coinciden y se completan al señalar la permanencia de esta villa durante un largo período.

Si tuviésemos que destacar cual es la época de mayor esplendor, señalaríamos el siglo । D.C., probablemente la época de su fundación, por la abundancia e importancia de los materiales recogidos, a pesar de no haberse hallado ninguna moneda perteneciente al mismo. $Y$ finalmente, tendríamos una segunda etapa de reactivación en el siglo IV, coincidiendo este período con el mayor número de monedas aparecidas y entroncando este momento con otro yacimiento muy próximo, como es la villa y necrópolis romana de «El Albir», descubierta no hace mucho, que por lo que nos ha comunicado y adelantado el Sr. Morote ${ }^{19}$, corresponde también al siglo $\mathrm{V}$.

${ }^{19}$ G. Morote: «Alfaz del Pi, El Albir», Arqueología, 79, n. ${ }^{\circ} 11 ; 80, \mathrm{n} .{ }^{\circ} 8 ; 82$, n. ${ }^{\circ} 6 ; 83$, n. ${ }^{\circ} 16.3$; y “El Albir», Arqueología en Alicante (1976-1986), Alicante, 1986, págs. 57-60. 


\section{BIBLIOGRAFÍA}

Bruun, P. M.: The Roman Imperial Coinage, vol. VII, Londres, 1966.

Carson, R. A. G.; HILL, P. V. y Kent, J. P. C.: Late Roman Bronze Coinage, Londres, reimpresión en 1978. Se cita por LRBC.

COHEN, H.: Description historique des monnaies frappées sous l'Empire Romain. París-Londres, 1880 . Se cita por COHEN.

KENT, J. P. C.: The Roman Imperial Coinage, vol. VIII, Londres, 1981.

Mattingly, H.: Coins of the Roman Empire in the British Museum, vol. IV y V, Londres, 1968.

Robertson, A. S.: Roman Imperial Coins in the Hunter Coin Cabinet, vol.

IV, Glasgow, 1978. 